\title{
Acknowledgement to Referees
}

Published online: 8 November 2017

(C) Springer International Publishing AG, part of Springer Nature 2017

Dear Reader,

As we reach the final issue of Targeted Oncology for 2017, I wish to reflect on another successful year's achievements, and to thank all those who have contributed their time and effort to guarantee the high quality of our content over the past 12 months.

In 2017, 70 articles have been published in Targeted Oncology; the most popular of these, in terms of downloads from SpringerLink (as of October 2017), have been:

Florczuk M., Szpechcinski A., Chorostowska-Wynimko J. miRNAs as Biomarkers and Therapeutic Targets in Non-Small Cell Lung Cancer: Current Perspectives. Targeted Oncology (2017), 12(2):179-200.

Rosen L.S., Jacobs I.A., Burkes R.L. Bevacizumab in Colorectal Cancer: Current Role in Treatment and the Potential of Biosimilars. Targeted Oncology (2017), 12(5):599-610.

Kim E.S., Scott L.J. Palbociclib: A Review in HR-Positive, HER2-Negative, Advanced or Metastatic Breast Cancer. Targeted Oncology (2017), 12(3):373-383.

Dhillon S. Obinutuzumab: A Review in Rituximab-Refractory or -Relapsed Follicular Lymphoma. Targeted Oncology (2017), 12(2):255-262.

Gupta N., Yang H., Hanley M.J., et al. Dose and Schedule Selection of the Oral Proteasome Inhibitor Ixazomib in Relapsed/ Refractory Multiple Myeloma: Clinical and Model-Based Analyses. Targeted Oncology (2017), 12(5):643-654.

Keating G.M. Sorafenib: A Review in Hepatocellular Carcinoma. Targeted Oncology (2017), 12(2):243-253.

Skaltsa K., Ivanescu C., Naidoo S. et al. Adjusting Overall Survival Estimates after Treatment Switching: a Case Study in Metastatic Castration-Resistant Prostate Cancer. Targeted Oncology (2017), 12(1):111-121.

Bylicki O., Paleiron N., Margery J., et al. Targeting the PD-1/PD-L1 Immune Checkpoint in EGFR-Mutated or ALKTranslocated Non-Small-Cell Lung Cancer. Targeted Oncology (2017), 12(5):563-569.

Corallo S., D'Argento E., Strippoli A., et al. Treatment Options for EGFR T790M-Negative EGFR Tyrosine Kinase InhibitorResistant Non-Small Cell Lung Cancer. Targeted Oncology (2017), 12(2):153-161.

Wise-Draper T.M., Moorthy G., Salkeni M.A., et al. A Phase Ib Study of the Dual PI3K/mTOR Inhibitor Dactolisib (BEZ235) Combined with Everolimus in Patients with Advanced Solid Malignancies. Targeted Oncology (2017), 12(3):323-332.

These 10 articles alone have been downloaded almost 8,000 times all together, while all articles published in 2017 were downloaded an impressive 22,000 times (as of October 2017).

The quality of articles published in the journal is also reflected in its most recent impact factor 3.438, making 2016 the fourth consecutive year with an impact factor over 3 . 
Other journals in the Adis Premier Journals Portfolio (http://www.springer.com/gp/adis) continue to prosper, and this year has seen the successful launch of PharmacoEconomics - Open, the newest journal in the portfolio. The journal has published a full volume of 30 articles in its first year, plus many more online first. Following in the steps of Adis' flagship subscription journal in the field, PharmacoEconomics, the new open-access journal focuses on cost and health outcomes associated with drugs, devices and other healthcare interventions.

We thank the authors who have contributed articles to Targeted Oncology over the course of 2017. The skill and dedication of all authors are critical to the continued publication of the journal. The quality of published articles is also testament to the significant efforts of the peer reviewers, whose commitment ensures that the journal's content is held to the highest possible standard. We would like to thank the following individuals who acted as reviewers for Targeted Oncology in the last 12 months:

Elisabetta Abruzzese, Italy

Sarah Adams, USA

Per Albertsson, Sweden

Aref Al-Kali, USA

Maria Alsina, Spain

Ajjai Alva, USA

Annarosa Arcangeli, Italy

Jean Bernard Auliac, France

Maximilian Bösch, Switzerland

Jorge Barriuso, UK

Debasish Basak, USA

Alexandr Bazhin, Germany

Andrea, Becchetti, Italy

Jens Bedke, Germany

Line Bjørge, Norway

Cherie Blenkiron, New Zealand

Jan Bouchal, Czech Republic

Massimo Breccia, Italy

Lieve Brochez, Belgium

Barna Budai, Hungary

Sebastiano Buti, Italy

Paolo Capogrosso, Italy

Paul Carroll, UK

Beatriz Carvalho, the Netherlands

Pedro Castelo-Branco, Portugal

Daniel Catenacci, USA

Joseph Chao, USA

Zirong Chen, USA

Katherine B. Chiappinelli, USA

Alberto A. Chiappori, USA

Rita Chiari, Italy

Christos Chouaid, France

Pippa Corrie, UK

Maria Di Bartolomeo, Italy

Giovan Giuseppe Di Costanzo, Italy

Mark Dickson, USA

Palma Dileo, UK

Frede Donskov, Denmark

Julien Edeline, France

Basse El-Rayes, USA

Jérôme Fayette, France
Nicola Fazio, Italy

Laura Foj, Spain

Andres Forero, USA

Udo Gaipl, Germany

Xavier Garcia del Muro, Spain

Pere Gascon, Spain

Daniel Geynisman, USA

Sharlene Gill, Canada

Kathryn Gold, USA

Joao Goncalves, Portugal

Alvaro González, Spain

Michael Gough, USA

William J. Gradishar, USA

Elin Gray, Australia

Valerie Grignol, USA

Petros Grivas, USA

Andrei Gudkov, USA

Shilpa Gupta, USA

Sarah Gwynne, UK

Dieter Hörsch, Germany

Balazs Halmos, USA

Peter Hawkins, USA

Hidetoshi Hayashi, Japan

Andrew Hendifar, USA

Miyoshi Hiroaki, Japan

Vera Hirsh, Canada

Robert R.A. Huddart, UK

David H. Ilson, USA

Eiji Iwama, Japan

Byoung Kuk Jang, Republic of Korea

Filip Janku, USA

Terrance Johns, Australia

Philip Johnson, UK

Ian R. Judson, UK

Wojciech Jurczak, Poland

Andrea Kasinski, USA

Keisuke Katsushima, Japan

Michael W. Kattan, USA

Ganessan Kichenadasse, Australia

Kyoung-Mee Kim, Republic of Korea

David Kroeger, Canada 
Geoffrey Y. Ku, USA

Makoto Kubo, Japan

Shaji K. Kumar, USA

Ruprecht Kuner, Germany

Athanassios Kyrgidis, Greece

Sara López-Tarruella, Spain

Justin Lathia, USA

Nathan Lawrentschuk, Australia

Richard J. Lee, USA

Herve Lena, France

Suzanne Lentzsch, USA

Konstantinos Leventakos, USA

Henk Lokhorst, the Netherlands

Florian Lordick, Germany

Xiaoming Lyu, China

Yuk Ting Ma, UK

Urs Müller-Richter, Germany

Stephan Macher-Goeppinger, Germany

Georgios Margonis, USA

Yoshiro Maru, Japan

María-Victoria Mateos, Spain

Benjamin Maughan, USA

Felicity May, UK

David F. McDermott, USA

Stephen McQuaid, UK

Andrew L. Mellor, UK

Taha Merghoub, USA

Michael Michael, Australia

Massimo Milione, Italy

Dominik Modest, Germany

Peter L. Molloy, Australia

Yong Wha Moon, Republic of Korea

Roger Moorehead, Canada

Alessandro Morabito, Italy

Teresa Moran, Spain

John Charles Morris, USA

Anita Muthukaruppan, New Zealand

Masahito Nakano, Japan

Ajay K. Nooka, USA

Kaname Nosaki, Japan

Takahiro Ochiya, Japan

Enrique M. Ocio, Spain

Timothy O'Connor, USA

Walter Olson, USA

Nader Omidvar, UK

Egbert Oosterwijk, the Netherlands

Albert Oriol, Spain

Gregory A. Otterson, USA

Luis Pardo, Germany

Jai Patel, USA

Paola Patrignani, Italy

Katriina Peltola, Finland

Maurice Perol, France
Ruth Pettengell, UK

Francesca Pettinella, USA

Paulo Pinheiro, USA

Maria Pia Pistillo, Italy

Lisa A. Porter, Canada

Jean-Pierre Pouget, France

Giuseppe Procopio, Italy

Andrew Protheroe, UK

Richard Quek, Singapore

Luis Raez, USA

Camille Ragin, USA

Raajit Rampal, USA

Jean-Luc Raoul, France

Mattea Reinisch, Germany

Jordi Remon-Masip, Spain

Xiubao Ren, China

Eva Rettinger, Germany

Lorenza Rimassa, Italy

Tadeusz Robak, Poland

Vânia Roberto, Portugal

Joseph Rosenblatt, USA

Piotr Rutkowski, Poland

Shella Saint Fleur-Lominy, USA

Dario Sangiolo, Italy

Oliver O. Sartor, USA

Lydia Scarfo, Italy

Mario Scartozzi, Italy

Matthias Scheffler, Germany

Werner W. Scheithauer, Austria

Ulrike Schick, France

Roxana Schillaci, Argentina

Ingo Schmidt-Wolf, Germany

Anja Seckinger, Germany

Cristiana Sessa, Switzerland

Yan-Shen Shan, Taiwan, Republic of China

Chan Shen, USA

Hirotaka Shibata, Japan

Janet Shipley, UK

Alexander N. Shoushtari, USA

Ron Smits, the Netherlands

Elizabeth Smyth, UK

Howard Sofen, USA

Ross A. Soo, Singapore

Pierre P.J. Souquet, France

Janakiraman Subramanian, USA

Mitsukuni Suenaga, Japan

Inge Marie Svane, Denmark

Lukasz Michal Szafron, Poland

Pierosandro Tagliaferri, Italy

Daisuke Takahari, Japan

Shunji Takahashi, Japan

Haruhiko Takeda, Japan

Dean Tang, USA 
Sebastian Theurich, Germany

Gianluca Tomasello, Italy

Amanda Townsend, Australia

Andrea Trotti, USA

Junji Tsurutani, Japan

Stephanie C. Tucker, USA

Anton H. van den Meiracker, the Netherlands

Guy van Hazel, Australia

Jean-Luc Van Laethem, Belgium

Terence Van Raay, Canada

Marcel Verheij, the Netherlands

Norbert Vey, France

Umberto Vitolo, Italy

Semir Vranic, Bosnia and Herzegovina
Derek Wainwright, USA

Harald Wajant, Germany

Eunice Wang, USA

Feng Wang, USA

Florian Weissinger, Germany

Steven Whittaker, UK

Jürgen Wolf, Germany

Xiaoliang Wu, USA

J. Yamaguchi, Japan

Muh-Hwa Yang, Taiwan, Republic of China

Haihong Yang, China

Chun-Nan Yeh, Taiwan, Republic of China

T. Yuasa, Japan

Quan Zhang, China

In addition, we would like to thank the members of the journal's Honorary Editorial Board, who have acted as peer reviewers and authors, and have provided guidance on journal content, policy and processes:

James O. Armitage, USA

James R. Berenson, USA

Jean-Yves Blay, France

Tomas Buchler, Czech Republic

Paul B. Chapman, USA

Ann-Lii Cheng, Taiwan

Fortunato Ciardiello, Italy

Jayesh Desai, Australia

Sandrine Faivre, France

Masahiro M. Fukuoka, Japan

Giuseppe Giaccone, USA

F. Anthony Greco, USA

Cesare Gridelli, Italy

Gabriel N. Hortobagyi, USA

V. Craig Jordan, USA

John M. Kirkwood, USA

Heinz-Josef Lenz, USA

Raymond Liang, Hong Kong

Teresa Macarulla, Spain

William P. McGuire, USA
Mark J. McKeage, New Zealand

Alain Mita, USA

Monica Mita, USA

Jean-François Morère, France

Athanasios G. Papavassiliou, Greece

Solange Peters, Switzerland

Camillo C. Porta, Italy

Shukui Qin, China

Eric Raymond, France

Olivier Rixe, USA

Christian Rolfo, Belgium

Matteo Santoni, Italy

Hans-Joachim Schmoll, Germany

Nicole A. Shonka, USA

Denis Soulières, Canada

Josep Tabernero, Spain

Giampaolo Tortora, Italy

Eric Van Cutsem, Belgium

John Eu Li Wong, Singapore

We do hope that you have found the articles published throughout the year in Targeted Oncology to be interesting and informative. The editorial schedule for 2018 is well under way, and we are looking forward to keeping you up to date with topical issues in the molecularly targeted oncology field in the next year.

With best wishes from

Martin Chopra

Editor

Targeted Oncology 